\title{
SISTEMA REPRODUTOR DE GUNDLACHIA CONCENTRICA (ORBIGNY) E GUNDLACHIA MORICANDI (ORBIGNY), COMO SUBSÍDIO PARA A TAXONOMIA DE ANCYLIDAE (MOLLUSCA, GASTROPODA, PULMONATA)
}

\author{
Fernanda Pires Ohlweiler ${ }^{1}$ \\ Inga Ludmila Veitenheimer-Mendes ${ }^{2}$
}

\begin{abstract}
REPROIU (CTIVE SYSTIM OF (GUNDLACHLA CONCENTRICA (ORBIGNY) AND) GUNDLACHLA MORICANDI (ORBIGNY), AS SL BSIDY FOR THE TAXONOMY OF ANCYIIDAE: (Molluisea, Gastropot)a, Pilmonata). The reproductive system of (iundlachia concentrica (Orbigny, 1835) and (iundlachia moricandi (Orbigny. 1837) are deseribed. The following structures distinguished the specimens of those species: ovotestis, seminal vesicle, tubular prolongation of dorsoposterior region of uterus and duct of the copulation pouch.

KEY WORDS. Gastropoda. Gundlachia concentrica, Gundlachia moricandi. reproductive system, taxonomy
\end{abstract}

Gundlachia concentrica (Orbigny, 1835) e Gundlachia moricandi (Orbigny, 1837) são freqüentemente encontradas em ambientes límnicos do Estado do Rio Grande do Sul, Brasil.

A respeito da morfologia interna, dos trabalhos que citam $G$. concentrica e G. moricandi, destacam-se as publicações de HubENDick (1964) e MiQuel (1988). HuBENDiCK (1964) descreve e analisa algumas estruturas anatômicas de diferentes espécies de Ancylidae, dentre as quais encontram-se G. concentrica e G. moricandi. As estruturas anatômicas ressaltadas, por HuBENDICK (1964), auxiliam na determinação dos ancilídeos mais a nível de gênero, tornando-se difícil utilizá-las para separar as espécies. MIQUEL (1988) não encontra diferenças morfo-anatômicas entre o complexo peniano de G. concentrica e G. moricandi.

Visando definir caracteres que possam ser utilizados como elementos diagnósticos à nível específico, realiza-se o estudo morfo-anatômico do sistema reprodutor destas duas espécies de Ancylidae.

\section{MATERIAL E MÉTODOS}

Espécimens de G. concentrica e G. moricandi foram coletados em outubro de 1993, no Parque COPESUL de Proteção Ambiental, Triunfo (Rio Grande do

1) Laboratório de Biologia Celular, Instituto Butantan. Av. Vital Brasil 1500. Caixa Postal 65, 05503-900 São Paulo. São Paulo. Brasil.

2) Departamento de Zoologia. Instituto de Biociêneias da Universidade Federal do Rio Grande do Sul, 90040-060 Porto Alegre, Rio Grande do Sul. Brasil. 
Sul): no açude (12 ex., MCN 33826) e no rio Caí (70 ex., MCN 33822), respectivamente. Gundlachia concentrica foi obtida também, em julho de 1993, no açude do Morro Santana, Porto Alegre (Rio Grande do Sul) (100 ex., MCN 33512).

Para o estudo morfológico do sistema reprodutor, os espécimens, foram anestesiados com thionembutal. No exame microanatômico, os animais, previamente anestesiados, foram fixados em formol $10 \%$ e incluídos em parafina. Foram realizados cortes histológicos seriados transversais para a contagem do número de lóbulos do ovoteste; e cortes longitudinais para a observação das demais estruturas. Os cortes de 7 um de espessura foram corados com hematoxilina de Harris e eosina.

A nomenclatura utilizada baseia-se em OHLwEILER \& LANZER (1994).

O material encontra-se depositado no Museu de Ciências Naturais da Fundação Zoobotânica do Rio Grande do Sul (MCN).

\section{RESULTADOS E DISCUSSÃO}

O sistema reprodutor (Figs 1-5) é hermafrodita. O ovoteste (OV) apresenta de 24 a 25 lóbulos em $G$. concentrica, número inferior ao de $G$. moricandi, que possui 30 a 38 lóbulos. Os lóbulos do ovoteste desembocam no átrio (AT), a partir do qual segue o ducto hermafrodita (DH). A vesícula seminal (VS) é constituída por divertículos, sendo que em $G$. concentrica estão espaçados e distribuídos ao longo da região mediana do ducto hermafrodita e em $G$. moricandi encontram-se concentrados em uma reduzida área do ducto, próximo ao átrio, assemelhando-se à fïgura feita, para esta espécie, por HuBENDICK (1964: 35, fig. 121). O ducto hermafrodita desemboca no carrefour, separando-se no oviducto e espermioducto. $\mathrm{Na}$ região do carrefour está inserida a bolsa de fertilização (BF) que se conecta com o oviducto e a glândula do albume (GA). O oviducto continua no útero, o qual é formado pela glândula nidamental $(\mathrm{GN})$, região anterior (RA) e região dorso-posterior (RDP). A região dorso-posterior do útero forma uma prolongação tubular (PT) na porção posterior esquerda, sendo que em $G$. concentrica esta prolongação é longa e com a extremidade livre dilatada, enquanto que em $G$. moricandi é curta, estreita e com a extremidade livre não dilatada. A ilustração do sistema reprodutor de G. moricandi apresentada por FERNANDEZ (1981: lâm. 2, fig. 1) mostra a prolongação tubular da região dorso-posterior do útero tal como aqui descrita. A bolsa da copulação (BC) conecta-se com a vagina (V) através de um ducto (DB), sendo que em $G$. concentrica este ducto é longo, ultrapassando o comprimento da vagina; enquanto que em $G$. moricandi é curto, medindo aproximadamente a metade do comprimento da vagina. HuBENDick (1964) também menciona o ducto da bolsa da copulação curto em $G$. moriandi, não havendo nenhuma observação para $G$. concentrica. O espermioducto chega à próstata $(\mathrm{PR})$, continuando-se pelo ducto deferente (DD). G. concentrica apresenta a próstata constituída por cinco a sete lóbulos e G. moricandi por seis a oito lóbulos. O ducto deferente insere-se no órgão copulador, o qual está constituído por um flagelo $(\mathrm{F})$, bainha (B), ducto ejaculatório (DE) e prepúcio (P). O flagelo, longo e glandular, contém um ducto (DF) em seu interior; o ducto ejaculatório abre-se na cavidade do prepúcio através de uma projeção tubular (PRT), também observada por 

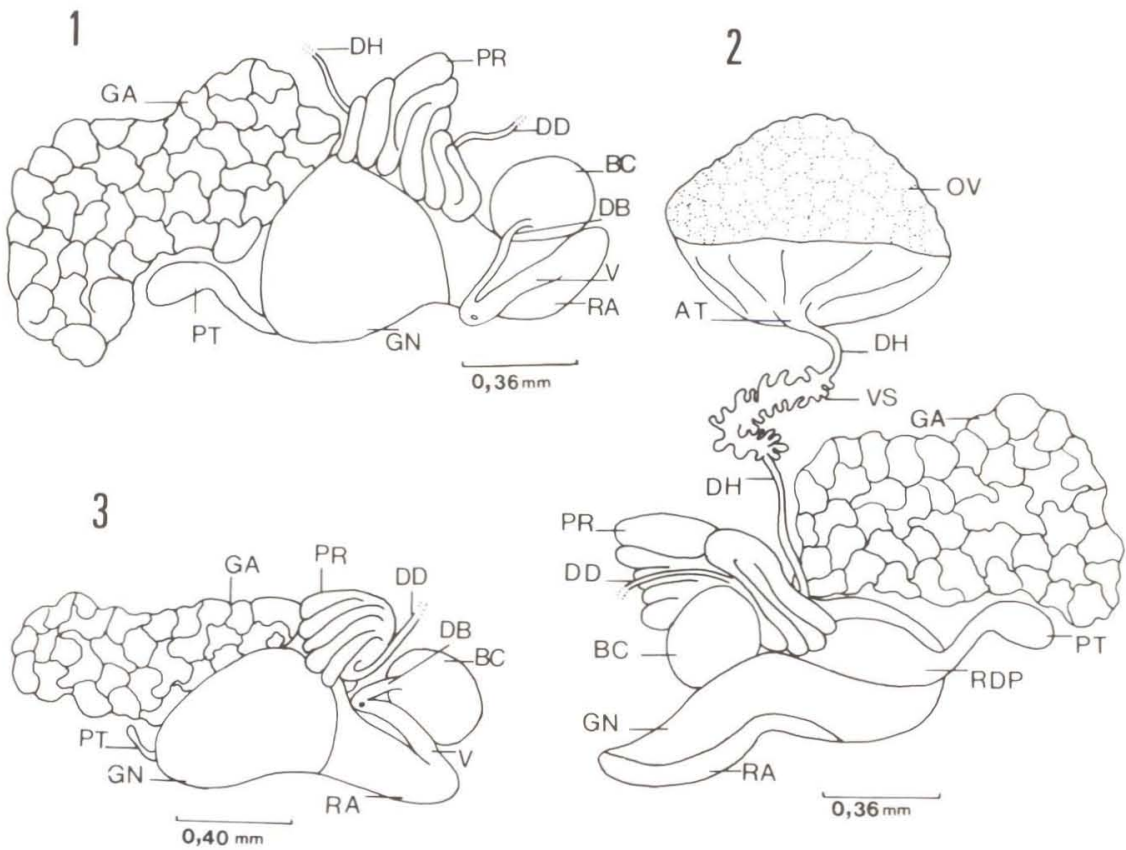

Figs 1-3. Sistema reprodutor. (1-2) Gundlachia concentrica; (3). Gundlachia moricandi. (AT) átrio, (BC) bolsa da copulação, (DB) ducto da bolsa da copulação, (DD) ducto deferente, (DF) ducto do flagelo, (DH) ducto hermafrodita, (GA) glândula do albume, (GN) glândula nidamental, (OV) ovoteste, (PR) próstata, (PT) prolongação tubular, (RA) região anterior do útero, (RDP) região dorso-posterior do útero, (V) vagina, (VS) vesícula seminal.

MiQuel (1988: 129, fïg. 1). Esta projeção tubular é denominada, por HuBENDICK (1964), de ponta evertida em G. concentrica e de uma espécie de ultra-pênis funcional em G. moricandi. Segundo HuBENDicK (1964) o ultra-pênis é uma estrutura formada por uma fenda na bainha e pode ser observada em algumas espécies de Laevapex Walker, 1903. O órgão copulador das espécies de Gundlachia Pfeiffer, 1949 é destituído de ultra-pênis e pênis. HUBENDICK (1955) afirma que a inserção do ducto ejaculatório na cavidade do prepúcio compensa a ausência de um pênis. Portanto, como se trata de uma estrutura distinta do ultra-pênis mantêm-se a denominação, tal como sugere OHLWEILER \& LANZER (1994), de projeção tubular. Existe um espaço entre a parede do ducto ejaculatório e a bainha. Concorda-se com MiQuel (1988) que os órgãos copuladores de G. concentrica e G. moricandi não apresentam diferenças morfo-anatômicas.

\section{CONCLUSÕES}

A partir deste estudo conclui-se que Gundlachia concentrica e G. moricandi podem ser distingüidas entre si com relação: ao número de lóbulos do ovoteste; à distribuição e disposição dos divertículos da vesícula seminal no ducto hermafrodita; à forma e comprimento da prolongação tubular da região dorso-posterior 
do útero; ao comprimento do ducto da bolsa da copulação.

Conclui-se ainda que o órgão copulador de $G$. concentrica e $G$. moricandi apresenta a mesma configuração morfo-anatômica.
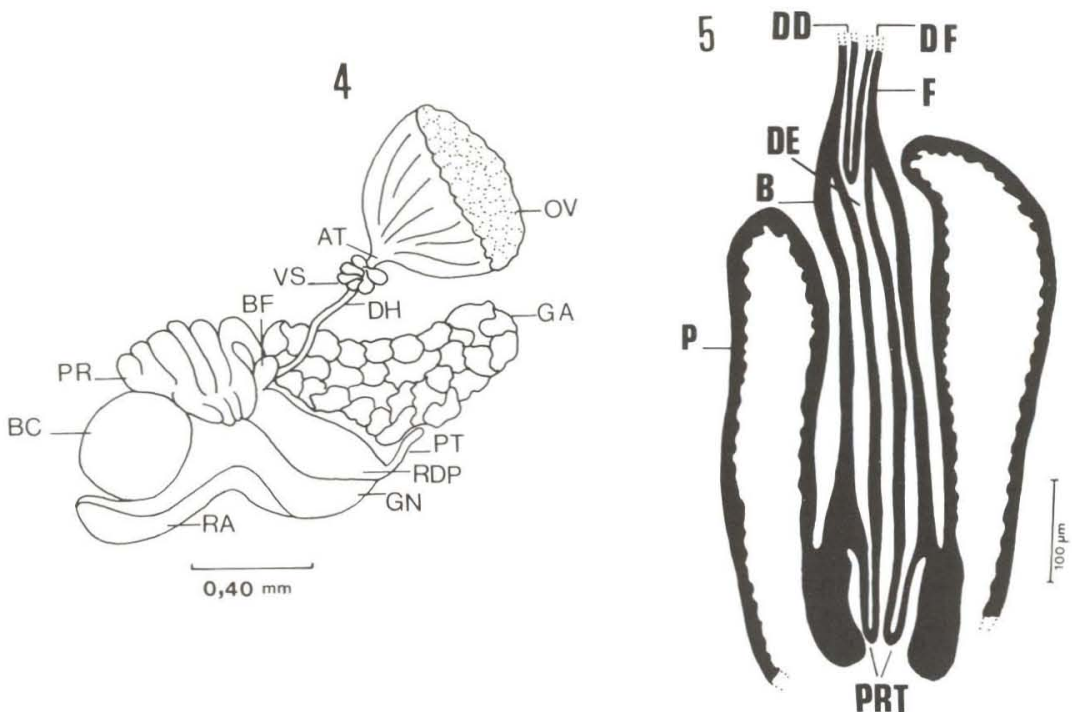

Figs 4-5. Sistema reprodutor. (4). Gundlachia moricandi; (5) corte longitudinal do órgão copulador de Gundlachia moricandi. (AT) átrio, (B) bainha, (BC) bolsa da copulação, (BF) bolsa de fertilização, (DD) ducto deferente, (DE) ducto ejaculatório, (DF) ducto do flagelo, (DH) ducto hermafrodita, (F) flagelo, (GA) glândula do albume, (GN) glândula nidamental, (OV) ovoteste, (P) prepúcio, (PR) próstata, (PRT) projeção tubular, (PT) prolongação tubular, (RA) região anterior do útero, (RDP) região dorso-posterior do útero, (VS) vesícula seminal.

\section{REFERÊNCIAS BIBLIOGRÁFICAS}

Fernandez, D. 1981. Molluscos Gasteropodos: Ancylidae. In: R.A. Ringuelet (ed.). Fauna de agua dulce de la republica Argentina. Buenos Aires, FECIC, 15 (7): 101-114.

HubENDICK, B. 1955. The anatomy of Gastropoda: The percy sladen trust expedition to lake Titicaca. Trans. Linnean Soc. London. 1 (3): 317-320.

- 1964. Studies on Ancylidae: The subgroups. Meddelanden Fran Goteborgs Musei, Zoologiska Avdelning, Goteborg, 137: 1-72.

MiQuel, S.E. 1988. Estudio anatomohistologico del complejo peniano de dos especies de Gundlachia Pfeiffer, 1849 (Mollusca, Pulmonata, Ancylidae).

Mem. Soc. Ci. Nat. La Sale, Caracas, 48 (129): 127-131.

OHLweIler, F.P. \& LANZER, R.M. 1994. Morfologia de Gundlachia obliqua (Broderip \& Sowerby, 1832) (Mollusca, Gastropoda, Ancylidae). Iheringia, Sér. Zool., Porto Alegre, (77): 113-127.

Recebido em 13.VII.1994; aceito em 04.X.1995. 